\title{
Attentional guidance by working memory differs by paradigm: An individual-differences approach
}

\author{
Emma Wu Dowd • Anastasia Kiyonaga • Tobias Egner • \\ Stephen R. Mitroff
}

Published online: 4 March 2015

(C) The Psychonomic Society, Inc. 2015

\begin{abstract}
The contents of working memory (WM) have been repeatedly found to guide the allocation of visual attention; in a dual-task paradigm that combines WM and visual search, actively holding an item in WM biases visual attention towards memory-matching items during search (e.g., Soto et al., Journal of Experimental Psychology: Human Perception and Performance, 31(2), 248-261, 2005). A key debate is whether such memory-based attentional guidance is automatic or under strategic control. Generally, two distinct task paradigms have been employed to assess memory-based guidance, one demonstrating that attention is involuntarily captured by memorymatching stimuli even at a cost to search performance (Soto et al., 2005), and one demonstrating that participants can strategically avoid memory-matching distractors to facilitate search performance (Woodman \& Luck, Journal of Experimental Psychology: Human Perception and Performance, 33(2), 363-377, 2007). The current study utilized an individualdifferences approach to examine why the different paradigms - which presumably tap into the same attentional construct-might support contrasting interpretations. Participants completed a battery of cognitive tasks, including two types of attentional guidance paradigms (see Soto et al., 2005; Woodman \& Luck, 2007), a visual WM task, and an operation span task, as well as attention-related self-report assessments. Performance on the two attentional guidance paradigms did not correlate. Subsequent exploratory regression analyses revealed that memory-based guidance in each task was differentially predicted by visual WM capacity for one paradigm, and by attentionrelated assessment scores for the other paradigm. The current results suggest that these two paradigms-which have
\end{abstract}

E. W. Dowd $(\bowtie) \cdot$ A. Kiyonaga $\cdot$ T. Egner $\cdot$ S. R. Mitroff Department of Psychology and Neuroscience, Center for Cognitive Neuroscience, Duke University, Box 90999, Durham, NC 27708, USA

e-mail: emma.wudowd@duke.edu previously produced contrasting patterns of performancemay probe distinct aspects of attentional guidance.

Keywords Attention · Working memory · Attentional capture

\section{Introduction}

Working memory (WM) and attention are typically considered distinct cognitive constructs, yet they frequently overlap (Awh \& Jonides, 2001; Gazzaley \& Nobre, 2012). For example, the active maintenance of items in visual WM can bias attention toward matching items in the environment, even at the expense of the current task goals (Soto, Hodsoll, Rotshtein, \& Humphreys, 2008). However, this bias is not always observed (e.g., Woodman \& Luck, 2007), generating debate about whether the coupling between WM and attention is involuntary or under strategic control. While differences in memory-based attentional guidance could be attributed to a complex set of task parameters, it is also possible that the various task paradigms that engender conflicting interpretations are actually tapping into different cognitive processes.

Studies of memory-based attentional guidance typically involve a visual search task while simultaneously maintaining a visual object in WM. The critical manipulation is whether the search target, distractors, or neither matches the stimuli in WM. In one paradigm (e.g., Soto, Heinke, Humphreys, \& Blanco, 2005), participants view a colored shape WM item (e.g., a blue circle) before performing a visual search for a slanted-line target among straight-line distractors (Fig. 1A). The WM item can reappear in the search display surrounding a target (valid), distractor (invalid), or fail to reappear at all (neutral). The visual search stimuli are thus distinct from, but embedded within, the class of stimuli that could match WM. 


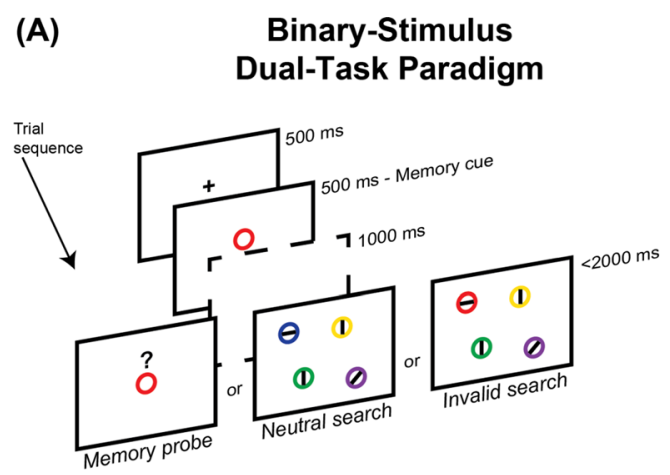

(C)

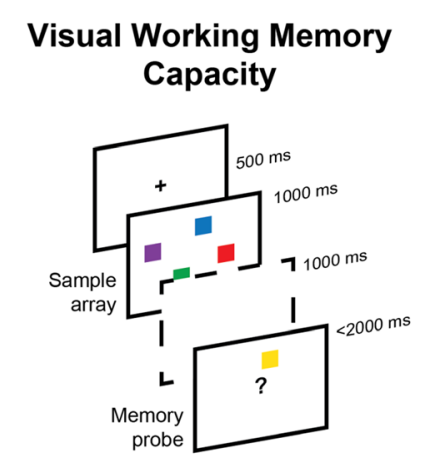

Fig. 1 Trial sequences for the four behavioral tasks. (A) Binary-stimulus dual-task paradigm that combines a memory task with an intervening search task (see Soto et al., 2005); participants searched for the tilted line target among vertical or horizontal line distractors. (B) Unitary-stimulus dual-task paradigm (see Woodman \& Luck, 2007); participants searched for the top or bottom gap target among right or left gap distractors. In both dual-task paradigms, the memory task tested whether the memory probe

The canonical finding is that search is speeded when the memory item validly cues the target and slowed when it invalidly cues a distractor (relative to the neutral condition; see Soto et al., 2008). Even when valid trials are eliminated from this task-de-incentivizing any strategic orienting toward the WM-match-search is still slowed on invalid trials (e.g., Kiyonaga, Egner, \& Soto, 2012; Olivers, 2009; Soto et al., 2005), suggesting the link between WM and visual attention is automatic (Soto et al., 2008; see also Dowd \& Mitroff, 2013; Hollingworth, Matsukura, \& Luck, 2013).

In contrast, other seemingly similar memory-based guidance tasks have produced different results. In one prominent task, participants view a colored square to hold in WM before searching among an array of colored Landolt C-like stimuli for a $\mathrm{C}$ whose gap is on the top or bottom (Woodman \& Luck, 2007; Fig. 1B). The search target or one of the distractors could match the WM color. In some studies using this task, visual attention was not captured by the memory-matching items (e.g., Han \& Kim, 2009; Woodman \& Luck, 2007). In fact, when there was no possibility of WM content matching

\section{(B) \\ Unitary-Stimulus \\ Dual-Task Paradigm}

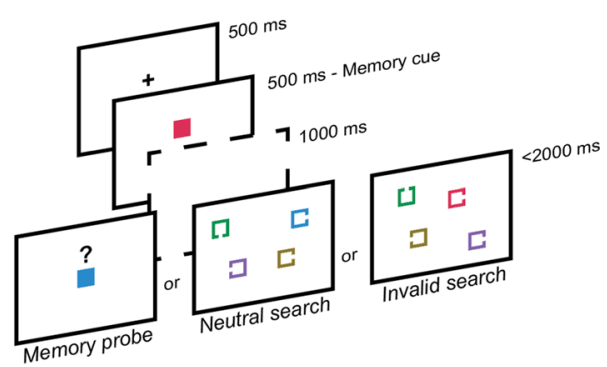

(D) Operation Span Task

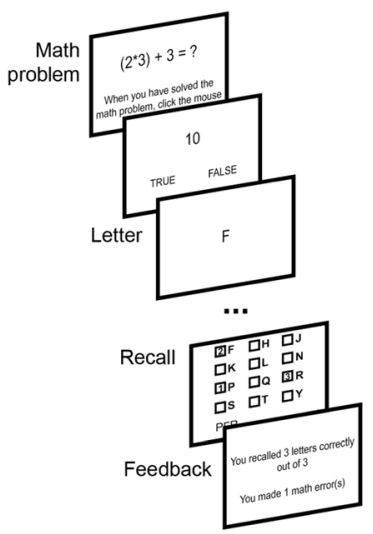

was same or different from the memory cue. (C) Visual working memory capacity task (see Luck \& Vogel, 1997); participants reported whether the memory probe color was same or different from the item at that location in the sample array. (D) Automated operation span task (Unsworth et al., 2005); participants alternated between remembering letters and solving arithmetic problems

the search target (i.e., no valid trials), there was a tendency for participants to be faster when a distractor matched WM, suggesting that participants were strategically avoiding the memory-matching distractors (Arita, Carlisle, \& Woodman, 2012; Woodman \& Luck, 2007, Experiment 3). These experiments suggest that there is no automatic visual selection bias toward WM-matching items, and that WM content can be strategically avoided (see Carlisle \& Woodman, 2011a).

Several studies have attempted to address why these superficially similar paradigms produce divergent results by focusing on specific task parameters that might underlie differences in memory-based guidance (e.g., Dalvit \& Eimer, 2011; Dombrowe, Olivers, \& Donk, 2010; Han \& Kim, 2009; Olivers, 2009; Soto \& Humphreys, 2008; Tsvetanov, Arvanitis, \& Humphreys, 2012; Woodman, Luck, \& Schall, 2007; Zhang et al., 2010, 2011). These investigations, however, have not coalesced into a unified picture of whether and how these factors impact memory-guided attention. Moreover, contradictory behavioral indices of memory-guided attention have been joined by contradictory electrophysiologic findings (i.e., 
differential modulation of the N2pc event-related potential component) from these two types of paradigms (Carlisle \& Woodman, 2011b, 2013; Kumar, Soto, \& Humphreys, 2009). Taken together, these findings challenge the assumption in this field that both types of paradigms probe the same underlying processes of how WM contents guide attention. Thus, one possible explanation for why two very similar paradigms engender different interpretations is that they are not measuring the same cognitive construct in the first place. This possibility has not been addressed, so the current study used an individual-differences approach to examine the relationship between performance on the two tasks. As a secondary focus, we also compare memory-based capture in each task relative to independently measured constructs of attention and WM.

\section{Methods}

\section{Participants}

Seventy-four individuals from Duke University (24 men, ages 18-27 years) participated for $\$ 10 /$ hour or course credit. All signed informed consent in accordance with institutional guidelines. Data were excluded from six additional participants who did not complete all four experimental tasks.

\section{Behavioral testing}

Participants completed four cognitive tasks (Fig. 1): two dualtask paradigms that combined WM and visual search (see Soto et al., 2005; Woodman \& Luck, 2007), a visual WM capacity task (see Luck \& Vogel, 1997), and a complex WM span task (automated operation span task; Unsworth, Heitz, Schrock, \& Engle, 2005). Visual WM capacity was included to reflect the strength of a WM representation (e.g., Bays \& Husain, 2008), which in turn could affect how memorymatching contents in the visual field capture attention. Complex span was included as a measure of WM maintenance in the face of interference, which resembles the dual-task setup of the attentional guidance paradigms in question here. Tasks were counterbalanced across participants, but the two dualtask paradigms were never completed consecutively.

Dual-task paradigms The two dual-task paradigms were based on designs by Soto and colleagues (Soto et al. 2005, 2008) and by Woodman \& Luck (2007; also see Carlisle \& Woodman, 2011a, 2011b; Han \& Kim, 2009). We refer to these tasks as using "binary stimuli" (when the memory-related circles and search-related lines have separate forms; see Soto et al., 2005) and "unitary stimuli" (when the memory-related color and the search-related gap exist within the same form; see Woodman \& Luck, 2007), respectively. We adapted the paradigms to have identical instructions, timing, display sizes, and stimulus colors; the contributions of these elements to memory effects on attention have been addressed by previous studies (e.g., Dombrowe et al., 2010; Olivers, 2009), and thus the current study focuses on the differences in stimulus arrangement. Importantly, the memory cue never matched the location of the search target, removing incentive to strategically look for the memorymatching search item (see Soto et al., 2005, Exp. 4).

Binary-stimulus paradigm In the binary-stimulus dual-task paradigm (Fig. 1A), each trial began with a 500-ms fixation display, followed by a 500-ms memory cue that was a colored circle $\left(1.3^{\circ} \times 1.3^{\circ}\right)$ centered at fixation. Participants were to remember the color of the cue for a potential future test. After a 1000-ms blank delay, a search array was presented until response (maximum presentation 2,000 ms). The search display consisted of four black lines ( $\operatorname{stroke}$ width $=0.2^{\circ}$, length $=1.0^{\circ}$ ), each surrounded by a colored circle. Three were horizontal or vertical, whereas the target line was tilted left or right at $45^{\circ}$. Each search item was placed with a slight spatial jitter within randomly selected cells of an invisible $5 \times 4$ grid subtending $10.3^{\circ} \times 8^{\circ}$, with no items overlapping. Each target location and orientation occurred equally often and in randomized order. Participants searched for the tilted line and reported the direction of the tilt via speeded keypress.

To prevent participants from strategically using the search array to refresh their memory of the memory item during the search task (see Woodman \& Luck, 2007), memory probes were given on $20 \%$ of trials instead of a search display. The memory probe was a colored circle $\left(1.3^{\circ} \times 1.3^{\circ}\right)$ presented at the center of the screen beneath a question mark for up to 2 , $000 \mathrm{~ms}$. Participants indicated whether the probe color was identical to the memory cue color via keypress. The memory cue, search circles, and memory probe were drawn randomly from five equidistant and equiluminant colors. In the search array, each color could only appear once.

Half of the search trials were neutral (memory item did not reappear) and half were invalid (memory item reappeared surrounding a distractor line). Memory probe trials were either a match $(50 \%)$ or a non-match $(50 \%)$ to the WM cue. After ten practice trials, participants completed 200 experimental trials.

Unitary-stimulus paradigm The unitary-stimulus dual-task paradigm was identical to the binary-stimulus task in timing and structure, but employed different stimuli (Fig. 1B). Memory cues and probes were colored squares $\left(0.65^{\circ} \times 0.65^{\circ}\right)$, and the search stimuli were outlined squares ( stroke width $=0.1^{\circ}$ ) with a $0.25^{\circ}$ gap on the top, bottom, left, or right. Three of the squares had a gap on the left or right, while the target square had a gap on the top or bottom. Participants searched for the top or bottom gap and reported the orientation of the gap via speeded keypress.

Visual working memory capacity The visual WM task (Fig. 1C) was adapted from Luck \& Vogel (1997). After a 
500-ms fixation display, a sample array of two, four, six, or eight colored squares $\left(1.0^{\circ} \times 1.0^{\circ}\right)$, jittered across a grid of $15.4^{\circ} \times 10.7^{\circ}$, was presented for $1,000 \mathrm{~ms}$. After a $1,000-\mathrm{ms}$ blank delay, a test array was presented, consisting of a single colored square in the same location as one of the sample array items. Participants reported whether the test item was identical to the sample item that had previously been shown in that location. Colors were pulled pseudorandomly from a circular spectrum of equiluminant colors. If the test item was different from the sample item, the difference was $180^{\circ}$ in color space.

Operation span The operation span task was administered with an automated version packaged by Unsworth et al. (2005). Participants were to remember information (sequences of unrelated letters, set sizes: 3-7) while concurrently engaging in online processing (solving arithmetic problems). On each trial participants alternated between solving math problems and memorizing single letters until a recall phase wherein they reported the letters in order (Fig. 1D). Each participant completed three sets of each set size (i.e., 15 sets). Full details of task structure and timing can be found in Unsworth et al. (2005).

\section{Individual-differences assessments}

Behavioral task data were collected along with a large battery of self-report assessments (see Appelbaum, Cain, Schroeder, Darling, \& Mitroff, 2012). The current study examined two attention-related surveys: the Attention Deficit-Hyperactivity Disorder (ADHD) questionnaire (Jasper \& Goldberg, 1993) and the Autism-Spectrum Quotient (ASQ; Baron-Cohen, Wheelwright, Skinner, Martin, \& Clubley, 2001) with a focus on the two attention-related sub-categories of attentionswitching and attention to detail. Both ADHD and autism have been linked to broader deficits in executive functions, including attentional control and WM (see Gargaro, Rinehart, Bradshaw, Tonge, \& Sheppard, 2011; Pennington \& Ozonoff, 1996), which could have important implications for memoryguided attention. No other questionnaire data were analyzed. ${ }^{1}$

\section{Results}

In the dual-task paradigms, our primary measure of interest was response time (RT) in the visual search task. Specifically, our key comparison was the time to find the target in neutral versus invalid conditions. To represent a single measure of attentional capture by WM, a normalized RT difference score was calculated for each individual: (invalid RT - neutral RT) / (neutral $\mathrm{RT}$ ), reflecting the absolute RT difference as a proportion of

\footnotetext{
${ }^{1}$ In line with previous research from our lab (e.g., Donohue, Darling, \& Mitroff, 2012), although we had a variety of self-report measures available, we only analyzed those that were identified a priori as relevant.
}

neutral RT. Positive values indicate high attentional capture, while negative values indicate reverse attentional capture.

Binary-stimulus paradigm

Performance on memory catch trials was nearly perfect $(M=99.1 \%, S D=1.7 \%)$. Search trials were excluded for response errors ( $0.7 \%$ of all search trials) and for exceeding the time limit $(0.4 \%$ of trials). RTs for correct search responses were slower for invalid trials $(M=1,045 \mathrm{~ms}, S D=138 \mathrm{~ms})$ than for neutral trials $(M=996 \mathrm{~ms}, S D=131 \mathrm{~ms}), t(73)=10.57$, $p<0.001$ (Fig. 2A). There was high variability across participants, with normalized RT difference scores $(M=0.05)$ ranging from +0.16 to -0.03 (for similar reports of individual variability, see Soto, Rotshtein, \& Kanai, 2014).

\section{Unitary-stimulus paradigm}

Performance on memory catch trials was nearly perfect $(M=98.6 \%, S D=2.7 \%)$. Search trials were excluded for response errors $(0.4 \%)$ and for exceeding the time limit $(1.0 \%)$. Search RTs for correct responses were slower for invalid trials $(M=1172 \mathrm{~ms}, S D=135 \mathrm{~ms})$ than neutral trials $(M=1128 \mathrm{~ms}$, $S D=136 \mathrm{~ms}$ ), $t(73)=7.08, p<0.001$ (Fig. $2 \mathrm{~A}$ ). There was high variability in normalized RT difference scores $(M=0.04)$ ranging from +0.15 to -0.09 . This particular paradigm has revealed variable capture effects (Carlisle \& Woodman, 2011b; Han \& Kim, 2009), and the data here demonstrated significant positive capture, in contrast to the reverse attentional capture effect from Woodman \& Luck (2007, Experiment 3$)^{2}{ }^{2}$ Importantly though, the presence or direction of a mean capture effect in this protocol is not a key concern of the purpose of the present study, which is to assess the relationship of individual differences in performance across tasks.

\section{Visual working memory capacity}

Visual WM capacity was calculated using Cowan's $K$ (Pashler, 1988; Cowan, 2001): $K=S(H-F)$, where $K$ is memory capacity, $S$ is set size, $H$ is observed hit rate, and $F$ is false

\footnotetext{
${ }^{2}$ It is possible that the specific timing and display differences between the current unitary-task paradigm and Woodman \& Luck's task may have engendered discrepant results. However, while both the binary-stimulus and unitary-stimulus tasks here produced positive capture, additional analyses did suggest differences in the magnitude of attentional capture. A Bayesian parameter estimation (Kruschke, 2013) using Markov chain Monte Carlo estimation was used to determine the likely distribution of attentional capture effect sizes in both paradigms. Attentional capture in the binary-stimulus paradigm had a mean effect size of $d=1.29$ [95\% HDI $0.96,1.62]$, whereas attentional capture in the unitary-stimulus paradigm was weaker, $d=0.89$ [95 \% HDI $0.59,1.19]$. In addition, search RTs for the unitary-stimulus paradigm were overall slower than RTs for the binary-stimulus paradigm, $F(1,73)=138.39, p<0.001$, likely due to the more perceptually demanding search task in the latter paradigm (Han \& Kim, 2009).
} 

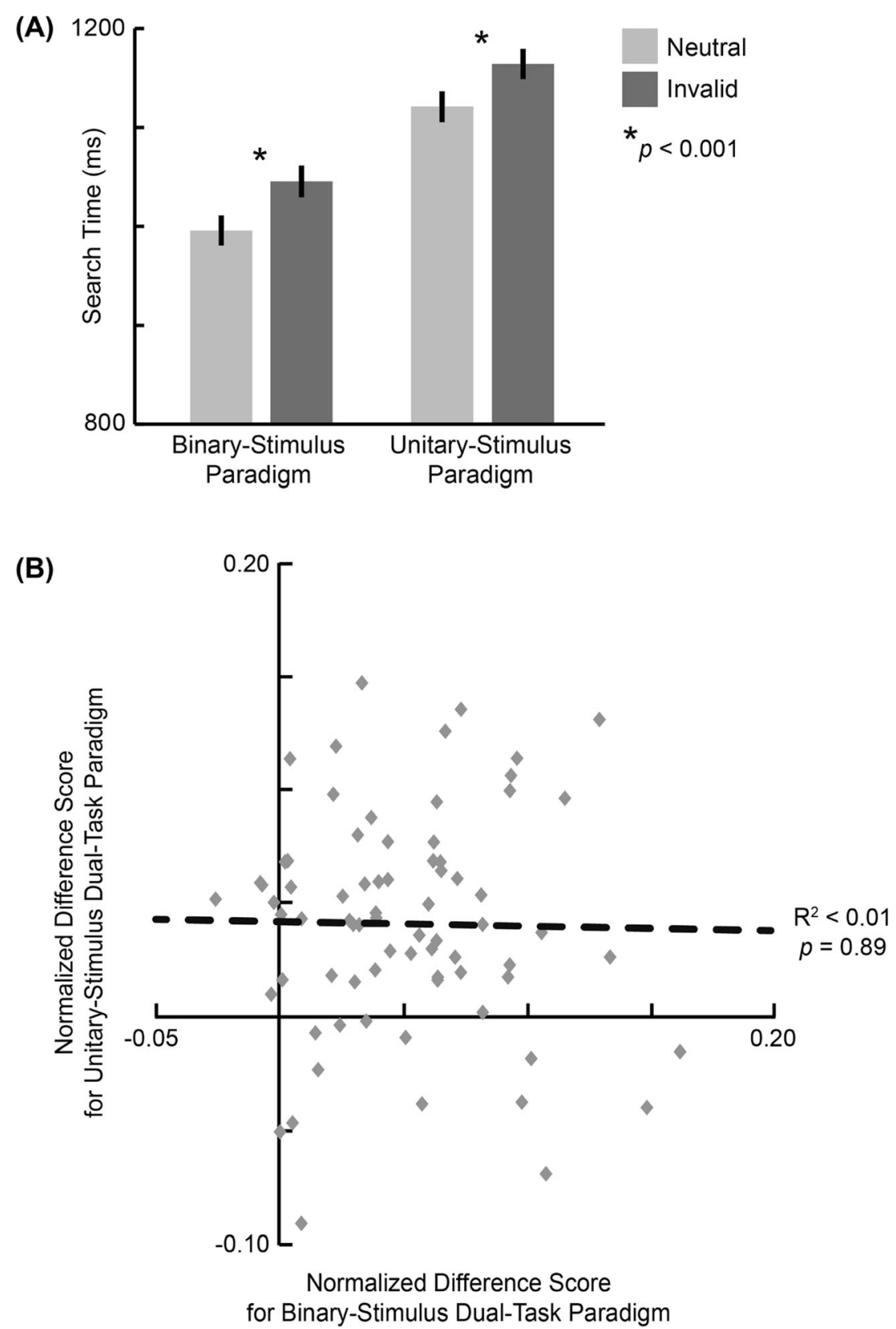

Fig. 2 (A) In both the binary-stimulus and unitary-stimulus paradigms, search times were slower for invalid trials compared to neutral trials. Error bars represent $95 \%$ confidence intervals. (B) Individual differences in

alarm rate. Final visual WM capacity was calculated by averaging $K$ values for set sizes 4, 6, and 8 (Fukuda \& Vogel, 2009). As in previous studies (e.g., Vogel \& Machizawa, 2004), mean WM capacity for our population was 3.20 $(S D=1.04)$, ranging from 1.05 to 5.39 .

\section{Operation span}

Operation span score was calculated by summing all perfectly recalled letter sets (see Unsworth et al., 2005). The mean operation span score was $49.2(S D=13.4)$, ranging from 20 to 75 . normalized difference scores (i.e., the amount of attentional capture by memory-matching distractors) did not correlate between the binarystimulus and unitary-stimulus paradigms

\section{Regression analyses}

Our analysis strategy was to use regression models to assess whether performance on the two dual-task paradigms was related. In a simple linear regression model, normalized difference scores on the binary-stimulus task did not significantly predict normalized difference scores on the unitary-stimulus task, adj. $R^{2}<0.001, F(1,72)=0.02, p=0.885,95 \%$ CI $[-0.20$, 0.17] (Fig. 2B). A Bayesian linear regression (Kruschke, 2011) revealed a posterior distribution of standardized slopes centered on $M=0.09$ [95\% HDI $-0.33,0.16]$, supporting the 


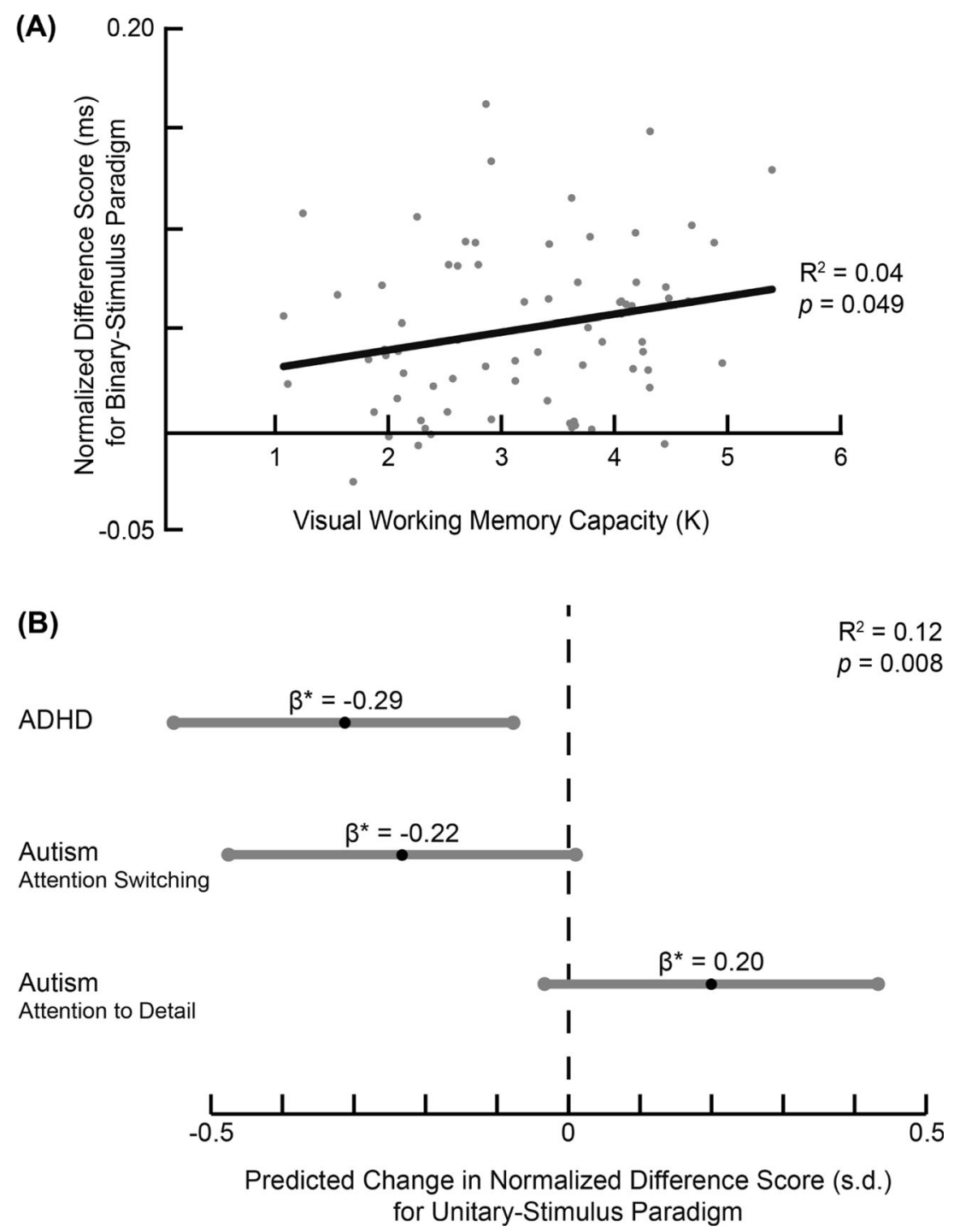

Fig. 3 Fitted linear regression models predicting memory-based capture in each dual-task paradigm by selected, significant variables of working memory and attention. (A) Normalized difference scores (i.e., the amount of attentional capture by memory-matching distractors) in the binarystimulus paradigm were significantly predicted by individual differences in visual working memory capacity. (B) Normalized difference scores in

conclusion that performance on binary-stimulus and unitarystimulus tasks were not significantly related. To show that this lack of correlation was not due to unreliability of either task, we used attenuation correction to adjust the correlation according to the reliability of the two measures being correlated (Schmidt \& Hunter, 1996). With difference score reliabilities of $\rho_{\text {binary }}=0.45$ and $\rho_{\text {unitary }}=0.41$, the corrected correlation between attentional capture effects in binary and unitary paradigms was still not significant, $r(74)=0.04, p=0.740 .^{3}$ To

\footnotetext{
${ }_{3}^{3}$ Although these reliability estimates seem low, it is important to note that difference scores are mathematically biased towards having relatively lower reliability than raw or composite scores (Webb, Shavelson, \& Haertel, 2006). For both paradigms, Cronbach's alpha (a measure of internal consistency) was $>0.95$ for both neutral and invalid trials.
}

the unitary-stimulus paradigm were significantly predicted by self-report assessments of attention deficit-hyperactivity disorder and two subcategories of the autism spectrum quotient. Lines depict the $95 \%$ confidence intervals around mean standardized regression coefficients (black dots) in predicting normalized difference scores in a multiple regression model

confirm that this lack of correlation was not due to validity effect differences between the tasks, search response times were subjected to a $2 \times 2$ repeated-measured ANOVA across validity and task; the interaction was non-significant, $F(1$, 73) $=0.51, p=0.479$.

The primary analysis of interest was the above correlation between attentional capture effects in the binary-stimulus and unitary-stimulus paradigms. Having found that they are not related to one another, we conducted exploratory regression analyses to see whether performance was differentially predicted by WM capacity or self-report measures of attention. A stepwise linear regression model was applied to both dual-task paradigms to explore what factors best predicted attentional capture for each. Five factors were included in an initial model 
for each paradigm: visual WM capacity, complex span, selfreport scores of ADHD, self-report scores on the attentionswitching subcategory of ASQ, and self-report scores on the attention-to-detail subcategory of ASQ.

In predicting performance for the binary-stimulus task, four factors (operation span, ADHD score, and two ASQ subcategory scores) did not significantly contribute to the model (full model, adj. $R^{2}=0.01$ ). Only visual WM capacity significantly predicted attentional capture, adj. $R^{2}=0.040, F(1$, $72)=3.99, p=0.049$ (Fig. 3A). Outliers were assessed based upon Cook's $D \geq 1$ (Cook \& Weisberg, 1982), and no data were trimmed.

In predicting performance for the unitary-stimulus task, two factors (visual WM capacity, operation span) did not significantly contribute to the model (full model, adj. $R^{2}=0.10$ ). Self-report scores of ADHD symptoms and the two ASQ subcategories significantly predicted attentional capture, adj. $R^{2}=0.119, F(3,70)=4.26, p=0.008$ (Fig. 3B). Again, no data points were trimmed for Cook's $D \geq 1$. No factor included in the model had a variance inflation factor above 1.12, indicating that collinearity was not an issue.

\section{General discussion}

A great number of studies have explored the extent to which WM content can capture visual attention, but debate remains open over whether attentional guidance by WM is automatic (e.g., Soto et al., 2008) or strategic (e.g., Woodman \& Luck, 2007). This debate has been fueled by conflicting findings from two seemingly similar dual-task paradigms, which putatively test the same aspect of attentional guidance. The current study employed an individual-differences approach to examine whether performance in one paradigm related to performance in the other. Attentional capture by WM in the binarystimulus (see Soto et al., 2005) and unitary-stimulus (see Woodman \& Luck, 2007) paradigms did not correlate, suggesting a fundamental difference in how each task taps into WM biasing, even within the same individuals.

Much of the evidence that has led to debate over memoryguided attention has been drawn from one or the other of these dual-task paradigms. On the surface, these paradigms seem very similar, generating the assumption that the two are assessing the same underlying phenomenon. In fact, at the group level, the current study observed capture effects of roughly the same magnitude across the two paradigms. Thus, it is all the more striking that the magnitude of attentional capture did not correlate at an individual level. The current behavioral results, along with previous contradictory electrophysiological findings between the two paradigms (see Carlisle \& Woodman, 2013), suggest the possibility that these two paradigms - which differ only in terms of stimulus arrangement - are actually tapping into distinct aspects of how memory and attention interact. The current results thus offer a new perspective on reconciling the disparate literature about memory-guided attention.

What might these distinct aspects of memory-based guidance entail? Using the other individual-differences measures, subsequent exploratory analyses hinted that attentional capture in the two paradigms was differentially predicted by independently measured constructs of WM and attention-specifically, by visual WM capacity for the binary-stimulus task, and by self-report assessments of attention for the unitarystimulus task. It is important to emphasize that the amount of variance explained by these factors was relatively small; furthermore, the current individual-differences approach did not allow for empirical manipulation of WM- and attentionrelated factors. Thus, these results only serve to generate initial hypotheses as to why the two paradigms produce different patterns of behavior.

The exploratory analyses revealed a dissociation between predictive factors for the binary-stimulus versus the unitarystimulus paradigm. For the binary-stimulus paradigm, greater visual WM capacity predicted greater attentional capture. Previous research has suggested that visual search processing taps into WM resources (e.g., Anderson, Vogel, \& Awh, 2013). We speculate that visual WM capacity might be more relevant for the binary-stimulus paradigm due to the physical distinction between binary and unitary stimuli. In binary-stimulus search arrays, there are two spatially distinct forms, the colored circle and the search line, which could theoretically be processed as separate "objects" (Duncan, 1984); in contrast, unitary stimuli consist of one form only. Thus, although only one "object" is encoded during the memory cue display, twice as many "objects" are processed in binary versus unitary search displays, which may tax WM resources to a greater degree.

Memory-based guidance in the unitary-stimulus paradigm was predicted by self-report assessments of ADHD and autism, which have been linked to difficulties with attentional control and flexibility (Geurts, Verté, Oosterlaan, Roeyers, \& Sergeant, 2004; Manly et al., 2001). These findings point to greater attentional capture in individuals who might be less able to flexibly deprioritize WM items (i.e., relegate them to accessory status; Olivers, Peters, Houtkamp, \& Roelfsema, 2011) during visual search. We speculate that these factors might be specifically predictive for performance in the unitary-stimulus paradigm due to the spatial concentration of task-relevant features, which may require more attentional control to dissociate the search-relevant feature (gap) from the memory-relevant feature (color). This issue goes handin-hand with search difficulty, in the sense that distinguishing between memory-relevant and task-relevant features in unitary stimuli is more perceptually demanding. However, general search difficulty in and of itself has not been found to influence memory-based attentional capture (Olivers, 2009). Overall, these exploratory analyses present a preliminary 
window into how the spatial differences between binary and unitary stimuli might differentially recruit object-encoding and attentional control processes, which may underlie the differences in attentional capture between the binary-stimulus and unitary-stimulus paradigms.

In much of the memory-guided attention literature, there has been an assumption that two superficially very similar and highly influential tasks are testing the same relationship between WM and attention, thus driving a debate about why results differ. By exploring these differences within the same individuals, the present study offers an alternative explanation: these two tasks produce non-correlating effects because they might be tapping into distinct aspects of memory-based attentional guidance. These findings underscore the importance of stimulus attributes and task design in determining the recruitment of particular attention and memory processes, and reveal that a multitude of cognitive factors can contribute to and influence the interaction between memory and attention.

Acknowledgments This work was partially supported by the Army Research Office (Grant No. 54528LS) and partially supported through a subcontract with the Institute for Homeland Security Solutions, a research consortium sponsored by the Resilient Systems Division in the Department of Homeland Security (DHS). This material is based on work supported by the DHS under Contract No. HSHQDC-08-C-00100. Any opinions, findings, and conclusions or recommendations expressed in this material are those of the authors and do not necessarily reflect the official policy or position of DHS or of the US Government. We thank Elise Kechele, Paul Skiba, and Eliza Gentzler for collecting data.

\section{References}

Anderson, D. E., Vogel, E. K., \& Awh, E. (2013). A common discrete resource for visual working memory and visual search. Psychological Science, 24(6), 929-938.

Appelbaum, L. G., Cain, M. S., Schroeder, J. E., Darling, E. F., \& Mitroff, S. R. (2012). Stroboscopic visual training improves information encoding in short-term memory. Attention, Perception, \& Psychophysics, 74(8), 1681-1691.

Arita, J. T., Carlisle, N. B., \& Woodman, G. F. (2012). Templates for rejection: configuring attention to ignore task-irrelevant features. Journal of Experimental Psychology: Human Perception and Performance, 38(3), 580-584.

Awh, E., \& Jonides, J. (2001). Overlapping mechanisms of attention and spatial working memory. Trends in Cognitive Sciences, 5(3), 119126.

Baron-Cohen, S., Wheelwright, S., Skinner, R., Martin, J., \& Clubley, E. (2001). The autism-spectrum quotient (AQ): Evidence from Asperger syndrome/high-functioning autism, males and females, scientists and mathematicians. Journal of Autism and Developmental Disorders, 31(1), 5-17.

Bays, P. M., \& Husain, M. (2008). Dynamic shifts of limited working memory resources in human vision. Science, 321(5890), 851-854.

Carlisle, N. B., \& Woodman, G. F. (2011a). Automatic and strategic effects in the guidance of attention by working memory representations. Acta Psychologica, 137(2), 217-225.
Carlisle, N. B., \& Woodman, G. F. (2011b). When memory is not enough: Electrophysiological evidence for goal-dependent use of working memory representations in guiding visual attention. Journal of Cognitive Neuroscience, 23(10), 2650-2664.

Carlisle, N. B., \& Woodman, G. F. (2013). Reconciling conflicting electrophysiological findings on the guidance of attention by working memory. Attention, Perception, \& Psychophysics, 75(7), 13301335.

Cook, R. D., \& Weisberg, S. (1982). Residuals and influence in regression. London: Chapman and Hall.

Cowan, N. (2001). The magical number 4 in short-term memory: a reconsideration of mental storage capacity. Behavioral and Brain Sciences, 24, 87-185.

Dalvit, S., \& Eimer, M. (2011). Memory-driven attentional capture is modulated by temporal task demands. Visual Cognition, 19(2), $145-153$.

Dombrowe, I., Olivers, C. N. L., \& Donk, M. (2010). The time course of working memory effects on visual attention. Visual Cognition, 18(8), 1089-1112.

Donohue, S. E., Darling, E. F., \& Mitroff, S. R. (2012). Links between multisensory processing and autism. Experimental Brain Research, 222(4), 377-387.

Dowd, E. W., \& Mitroff, S. R. (2013). Attentional guidance by working memory overrides salience cues in visual search. Journal of Experimental Psychology: Human Perception and Performance, 39(6), 1786-1796.

Duncan, J. (1984). Selective attention and the organization of visual information. Journal of Experimental Psychology: General, 113(4), 501.

Fukuda, K., \& Vogel, E. K. (2009). Human variation in overriding attentional capture. The Journal of Neuroscience, 29(27), 8726-8733.

Gargaro, B. A., Rinehart, N. J., Bradshaw, J. L., Tonge, B. J., \& Sheppard, D. M. (2011). Autism and ADHD: How far have we come in the comorbidity debate? Neuroscience \& Biobehavioral Reviews, 35(5), 1081-1088.

Gazzaley, A., \& Nobre, A. C. (2012). Top-down modulation: Bridging selective attention and working memory. Trends in Cognitive Sciences, 16(2), 129-135.

Geurts, H. M., Verté, S., Oosterlaan, J., Roeyers, H., \& Sergeant, J. A. (2004). How specific are executive functioning deficits in attention deficit hyperactivity disorder and autism? Journal of Child Psychology and Psychiatry, 45(4), 836-854.

Han, S. W., \& Kim, M. (2009). Do the contents of working memory capture attention? Yes, but cognitive control matters. Journal of Experimental Psychology: Human Perception and Performance, 35(5), 1292-1302.

Hollingworth, A., Matsukura, M., \& Luck, S. J. (2013). Visual working memory modulates rapid eye movements to simple onset targets. Psychological Science, 24(5), 790-796.

Jasper, L., \& Goldberg, I. (1993). Jasper/Goldberg adult ADD questionnaire.

Kiyonaga, A., Egner, T., \& Soto, D. (2012). Cognitive control over working memory biases of selection. Psychonomic Bulletin \& Review, 19(4), 639-646.

Kruschke, J. K. (2011). Bayesian assessment of null values via parameter estimation and model comparison. Perspectives on Psychological Science, 6(3), 299-312.

Kruschke, J. K. (2013). Bayesian estimation supersedes the $t$-test. Journal of Experimental Psychology: General, 142(2), 573-603.

Kumar, S., Soto, D., \& Humphreys, G. W. (2009). Electrophysiological evidence for attentional guidance by the contents of working memory. European Journal of Neuroscience, 30(2), 307-317.

Luck, S. J., \& Vogel, E. K. (1997). The capacity of visual working memory for features and conjunctions. Nature, 390(6657), 279-281.

Manly, T., Anderson, V., Nimmo-Smith, I., Turner, A., Watson, P., \& Robertson, I. H. (2001). The differential assessment of children's 
attention: The test of everyday attention for children (TEA-Ch), normative sample and ADHD performance. Journal of Child Psychology and Psychiatry, 42(8), 1065-1081.

Olivers, C. N. L. (2009). What drives memory-driven attention capture? The effects of memory type, display type, and search type. Journal of Experimental Psychology: Human Perception and Performance, 35(5), 1275-1291.

Olivers, C. N. L., Peters, J., Houtkamp, R., \& Roelfsema, P. R. (2011). Different states in visual working memory: When it guides attention and when it does not. Trends in Cognitive Sciences, 15(7), 327-334.

Pashler, H. (1988). Familiarity and visual change detection. Perception \& Psychophysics, 44(4), 369-378.

Pennington, B. F., \& Ozonoff, S. (1996). Executive functions and developmental psychopathology. Journal of Child Psychology and Psychiatry, 37(1), 51-87.

Schmidt, F. L., \& Hunter, J. E. (1996). Measurement error in psychological research: Lessons from 26 research scenarios. Psychological Methods, 1(2), 199-223.

Soto, D., \& Humphreys, G. W. (2008). Stressing the mind: The effect of cognitive load and articulatory suppression on attentional guidance from working memory. Perception and Psychophysics, 70(5), 924934.

Soto, D., Heinke, D., Humphreys, G. W., \& Blanco, M. J. (2005). Early, involuntary top-down guidance of attention from working memory. Journal of Experimental Psychology: Human Perception and Performance, 31(2), 248-261.

Soto, D., Hodsoll, J., Rotshtein, P., \& Humphreys, G. W. (2008). Automatic guidance of attention from working memory. Trends in Cognitive Sciences, 12(9), 342-348.
Soto, D., Rotshtein, P., \& Kanai, R. (2014). Parietal structure and function explain human variation in working memory biases of visual attention. NeuroImage, 89, 289-296.

Tsvetanov, K. A., Arvanitis, T. N., \& Humphreys, G. W. (2012). Dissociating effects of stimulus identity and load on working memory attentional guidance: Lengthening encoding time eliminates the effect of load but not identity. The Quarterly Journal of Experimental Psychology, 65(8), 1475-1483.

Unsworth, N., Heitz, R. P., Schrock, J. C., \& Engle, R. W. (2005). An automated version of the operation span task. Behavior Research Methods, 37(3), 498-505.

Vogel, E. K., \& Machizawa, M. G. (2004). Neural activity predicts individual differences in visual working memory capacity. Nature, 428(6984), 748-751.

Webb, N. M., Shavelson, R. J., \& Haertel, E. H. (2006). Reliability coefficients and generalizability theory. Handbook of Statistics, 26, 81-124.

Woodman, G. F., \& Luck, S. J. (2007). Do the contents of visual working memory automatically influence attentional selection during visual search? Journal of Experimental Psychology: Human Perception and Performance, 33(2), 363-377.

Woodman, G. F., Luck, S. J., \& Schall, J. D. (2007). The role of working memory representations in the control of attention. Cerebral Cortex, 17(suppl 1), i118-i142.

Zhang, B., Zhang, J. X., Kong, L., Huang, S., Yue, Z., \& Wang, S. (2010). Guidance of visual attention from working memory contents depends on stimulus attributes. Neuroscience Letters, 486(3), 202206.

Zhang, B., Zhang, J. X., Huang, S., Kong, L., \& Wang, S. (2011). Effects of load on the guidance of visual attention from memory. Vision Research, 51(23), 2356-2361. 